\title{
Alexithymia in parents and adolescent anorexic daughters: comparing the responses to TSIA and TAS-20 scales
}

This article was published in the following Dove Press journal:

Neuropsychiatric Disease and Treatment

9 October 2014

Number of times this article has been viewed

\author{
Laura Balottin' \\ Renata Nacinovich ${ }^{2}$ \\ Monica Bomba ${ }^{2}$ \\ Stefania Mannarini' \\ 'Interdepartmental Center of Family \\ Research, Department of Philosophy, \\ Sociology, Education, and Applied \\ Psychology, Section of Applied \\ Psychology, University of Padova, \\ Padova, Italy; ${ }^{2}$ Childhood and \\ Adolescence Neuropsychiatric Unit, \\ San Gerardo Hospital, University \\ of Milan-Bicocca, Monza, Italy
}

Background: A growing body of literature has been focusing on individual alexithymia in anorexia nervosa, while there are only scarce and conflicting studies on alexithymia in the families of anorexic patients, despite the important role played by family dynamics in the development of the anorexic disorder, especially in adolescent patients. The aim of this study is to assess alexithymia in anorexic adolescent patients and in their parents using a multimethod measurement to gain more direct, in-depth knowledge of the problem.

Methods: Forty-six subjects, anorexic adolescent patients and their parents, underwent the Toronto Alexithymia Scale (TAS-20) along with the Toronto Structured Interview for Alexithymia (TSIA), which represents the first comprehensive clinically structured interview focused specifically on assessing alexithymia. The use of latent trait Rasch analysis allowed a comparison of the two instruments' sensitivity and ability to detect the presence and intensity of alexithymic components in patients and parents.

Results: Significant discordance was found between the two measures. The clinical instrument allowed detection of a greater level of alexithymia compared with the self-report, in particular in our adult parent sample. Moreover, a significant alexithymic gap emerged within families, particularly within parental couples, with noticeably more alexithymic fathers compared with the mothers.

Conclusion: The TSIA clinical interview may be a more sensitive instrument in detecting alexithymia, minimizing parents' negation tendency. Clinical questions have arisen on how useful it would be to give greater weight to family functioning (ie, alexithymic gap) in order to predict the possibility of establishing a therapeutic alliance, and thus the outcome of the anorexic adolescent.

Keywords: anorexia, adolescence, family, Toronto Structured Interview for Alexithymia, Toronto Alexithymia Scale, Rasch model

\section{Introduction}

Anorexia is an eating disorder that generally affects adolescents, triggering severe relational imbalance to the family. The emotional functioning of families with an anorexic adolescent plays a pivotal role in the evolution of the disorder and in the therapies addressed not only to the adolescents but to the family as well. ${ }^{1}$ Nevertheless, still little is known about emotional functioning in this kind of family. Studies conducted on the general population have found a link between family pathological functioning and alexithymia, in particular between family emotional dysfunctions and difficulties identifying feelings. ${ }^{2}$ Despite the significance of this link, literature has focused greatly on individual alexithymia, producing only scarce and conflicting studies on alexithymia in families of anorexic patients..$^{3-5}$ Evidence, however, is now highlighting
Correspondence: Stefania Mannarini Department of Philosophy, Sociology, Education, and Applied Psychology, University of Padova, Via Venezia 8 35I3I Padova, Italy

Tel +390498276630

$\mathrm{Fax}+390498276600$

Email stefania.mannarini@unipd.it
Neuropsychiatric Disease and Treatment 2014:10 194I-195I 
the importance of family dynamics in the development of the anorexic disorder, ${ }^{6-8}$ especially in younger patients. ${ }^{1,9}$

Literature seems to confirm the link between use of body symptoms and difficulties in managing and expressing emotions at the symbolic verbal level. In fact, patients with eating disorders, anorexia in particular, reveal significantly higher alexithymic traits and difficulties in regulating affect. ${ }^{10-12}$ In first diagnosed anorexic adolescents, emotional dysregulation is associated with lower levels of diazepambinding inhibitor, a peptide involved in mood eating behavior regulation, ${ }^{13}$ and directly correlated to the severity of autonomic dysregulation. ${ }^{12}$

Difficulties in identifying feelings have proven to be an important prognostic factor in the evolution of anorexia. ${ }^{14}$ It must, however, be noted that research on the topic, conducted on adolescent samples, ${ }^{15-19}$ continues to be scarce, although this pathology typically arises in adolescence.

The emotional deficits of anorexic patients may be connected with their parents' emotional functioning. ${ }^{9}$ Studies on adult patients have yielded contradictory results. In Dahlman's ${ }^{3}$ first study, the mothers of anorexic patients resulted more alexithymic than controls, but this has not always been confirmed in subsequent studies. ${ }^{4}$ Furthermore, even in studies reporting higher levels of alexithymia in parents of an anorexic daughter, ${ }^{5}$ this alexithymic state seems mostly linked to considerable emotional distress, often linked to traumatic experiences. ${ }^{4,5}$

In general, studies conducted using self-report questionnaires (eg, the Toronto Alexithymia Scale [TAS-20]) show that the high levels of alexithymia reported by patients are often correlated to depression and negative affectivity. ${ }^{14,20-23}$ The high levels of alexithymia measured by self-report are not always confirmed by performance-based measures devised to assess the actual ability to attribute and express emotions..$^{24,25}$

This would trigger doubts on the validity and reliability of alexithymia self-administered measuring systems. Self-report instruments are inevitably influenced by the subject's will and ability to refer to what he or she feels, thus by the very capacity an alexithymic is not supposed to have. ${ }^{26-28} \mathrm{~A}$ more reliable assessment of such a complex construct as alexithymia would generally entail multimethod measuring ${ }^{29,30}$ that combines a categorial (diagnostic) approach as well as a dimensional (trait intensity) approach, attained using both self-administered and interviewer-administered instruments. An interesting study by Berthoz et $\mathrm{al}^{31}$ conducted on Eating Disorders (ED) patients has paved the way in this direction, comparing various alexithymia measuring instruments, among which we can find the widespread TAS-20 self-report and another, lesser known, report (Observer Alexithymia Scale d'Haviland ${ }^{32}$ ).

Considering the interesting results of the aforementioned study, the prime aim of this research was to use a multimethod measurement to assess alexithymia in anorexic adolescent patients and in their parents. For such a purpose two different instruments were chosen, a self-administered questionnaire and a structured clinical interview, TAS-20 33,34 and the Toronto Structured Interview for Alexithymia (TSIA). ${ }^{35}$ Both instruments developed by the Toronto-based group are theoretically similar, thus enabling a comparison of their sensitivity and ability to detect the presence and intensity of alexithymic components in families of anorexic patients.

The second aim of the study was to broaden the limited knowledge on alexithymia in adolescent anorexic patients and in their parents, considering them not only as isolated individuals but as members of an interconnected triad, a restricted family nucleus that has specific ways of expressing and managing feelings. We considered each triad as a restricted family nucleus in order to compare the alexithymia of the daughter, the mother, and the father and to determine the mean alexithymia score for each family nucleus.

\section{Materials and methods Participants}

The sample included 46 subjects: 16 anorexic adolescent patients (aged 13-17 years) and their 30 parents. The subjects were consecutive attendees at the Child and Adolescent Neuropsychiatric University Unit of the San Gerardo Hospital, University of Milan-Bicocca, Monza, Italy, from March to June 2013. We considered each triad (daughter, mother, father) as a restricted family nucleus. Due to ethical problems, siblings, where present, were excluded from this study, avoiding their medicalization. Participation in the study required that all participants gave their written informed consent. The study was carried out according to the standards for good ethical practice of San Gerardo Hospital.

Selection was based on the following inclusion criteria: restrictive Diagnostic and Statistical Manual of Mental Disorders, Fourth Edition, Text Revision (DSM IV-TR) diagnosis of anorexia nervosa, female, aged from 13 to 17 years, and parents' consent to participate in the study. Exclusion criteria instead included another major diagnosis (autism, schizophrenia, mental retardation) and undergoing psychotherapy for an extended period (more than 3 months).

The anorexic adolescent patients' mean age was 15.81 years, ranging from 13 to 17 years, and their mean current body 
mass index was 16 (standard deviation $=2$ ), resulting in being quite representative of the population usually attending the Child and Adolescent Neuropsychiatric University Unit and entering clinical research programs. ${ }^{12,13,19,36,37}$ Among the 32 parents, one of the fathers had passed away and one of the mothers could not be traced at the time of the research. The mothers' mean age was 45.8 years, ranging from 36 to 52 years, and fathers' mean age was 49.13 years, ranging from 38 to 61 years.

\section{Instruments}

\section{TAS-20}

The TAS- $20^{33,34}$ is the most widely used self-report questionnaire to measure alexithymia. This instrument provides the total score and also measures three major components of alexithymia: difficulty identifying feelings (F1), difficulty describing feeling to others (F2), and externally oriented thinking (F3).

There are 20 items, each evaluated using a five-point Likert scale, starting with 1 (strongly disagree) to 5 (strongly agree). The total score can vary from a minimum of 20 to a maximum of 100 . The Italian translation of the TAS-20 has demonstrated factorial validity, internal consistency, and high test-retest reliability. ${ }^{38}$

\section{TSIA}

The TSIA ${ }^{35}$ is an interview conceived to measure alexithymia. It comprises a total of 24 items subdivided into four subscales (each with six items) designed to evaluate four core dimensions of alexithymia: difficulty identifying feelings (DIF), difficulty describing feelings (DDF), externally oriented thinking (EOT), and imaginal processes (IMP).

Each question is scored on a three-point Likert scale ranging from 0 to 2 . Higher scores indicate a higher degree of alexithymia, and total scores range from 0 to 48 .
Its design stems from the need to compensate the weaknesses of the TAS-20 (Table 1): self-assessment and exclusion of a measure of poor imaginal processes. The presence of an external interviewer guarantees, contrarily to what happens with the self-report questionnaire, that the answer be investigated and clarified whenever the interviewee shows scarce consciousness of his/her problems.

The TSIA used along with the TAS-20 allows a multimethod assessment based on different measuring instruments, self and other report. ${ }^{30,39,40}$

In the validation study of the Toronto group,${ }^{35}$ as well as in the validation of the Italian version, ${ }^{41}$ the TSIA obtained a good interrater, test-retest, and internal reliability and concurrent validity with the TAS-20. The hierarchic factor structure is made up of four subscales grouped into two higher order factors: conscious emotional awareness (subscale 1 and 2) and operational thinking (subscale 3 and 4).

\section{Procedure}

A neuropsychiatrist with special interest and clinical experience in anorexia evaluated all patients, collecting a comprehensive family and medical history. Patients, diagnosed using the DSM-IV-TR criteria for restricting type anorexia nervosa, also completed the Eating Disorder Inventory (EDI-3). ${ }^{42}$ The diagnosis of comorbid disorders was supported by the Kiddie-SADS semistructured interview. ${ }^{43}$

Patients were asked to fill out the TAS-20 questionnaire and undergo the TSIA structured interview. Parents were asked to do the same. The setting remained the same for each patient and parent, each examined separately. The answers to the TSIA were collected by two specifically trained interviewers, both unaware of the scores obtained in the TAS-20 questionnaire. Interviews were then scored by two judges, one being an expert in using the instrument. In rare cases of disagreement in attributing the score, an agreement score was reached.

Table I Self-report versus clinical interview: advantages and disadvantages

Toronto Alexithymia Scale (TAS-20)

\section{Self-report}

Time required: 10-15 minutes

Quick to administer

Inexpensive

Simple to score

Assume patient is aware of his/her deficit

Assume subject's motivation to reply sincerely

Lack of a measure of imaginal processes

Limits due to respondent's motivation and ability

to reply sincerely
Toronto Structured Interview for Alexithymia (TSIA)

Structured interview

Time required: minimum 40 minutes

More laborious to administer

Training required to administer

Training required to score

Inquiry help clarifying the true nature of unclear responses

Presence of an external observer may provide a more valid

assessment

Assessment of fantasy activity

Accuracy depends on standardized administration and rating across different interviewers/raters 


\section{Analysis}

The study relied on the use of Rasch analysis. ${ }^{44}$ The Andrich Rating Scale Model ${ }^{45}$ was applied to estimate the measurements obtained from Likert type ratings provided by participants for each of the two scale items. The WINSTEP Ministep Rasch-Model program ${ }^{46}$ was used to estimate the level of alexithymia expressed by each person on the rating scale. Since the study's main aim is to compare the item responses ${ }^{47-51}$ provided by participants, Rasch analysis was applied to the data because of its psychometric features, in particular the invariance of comparisons property. As is generally known, when data fit the Rasch model properly and when comparing the responses of two people, such comparison is independent from stimuli or items that determine the responses. Rasch analysis was applied separately for the two scale total scores and for each of the common factors of the scales (factor 1, DIF; factor 2, DDF; factor 3, EOT). Furthermore, to compare the total scores and scale factors between the two scales, the estimates of each person were standardized. Subsequently, an alexithymia mean standard score was calculated for each family nucleus. To evaluate the difference between alexithymic levels of family members, a contrast standard value was calculated for each couple in the family, more specifically daughter versus mother, daughter versus father, and mother versus father. To summarize the data related to the 16 families, three categories, representing different levels of alexithymia, were created for the families and separately for daughters, mothers, and fathers: category 1 (low) included standard scores below $-1.64(P<0.05)$, thus respondents with low alexithymia levels; category 2 (middle) ranges between -1.64 and 1.64, thus with middle alexithymia levels; and category 3 (high) above $1.64(P<0.05)$, thus high alexithymia levels. Considering the contrasts between family members, the same categories were constructed. Denominating $\mathrm{A}$ and $\mathrm{B}$ the members of a couple, category 1 (low) included couples with statistically different levels of alexithymia, where $\mathrm{A}$ is less alexithymic than $\mathrm{B}$ (below -1.64 , $P<0.05$ ); category 2 (middle) included couples with statistically nonsignificantly different levels of alexithymia (between -1.64 and 1.64, $P>0.05$ ); and category 3 (high) contained couples with statistically different levels of alexithymia, where $\mathrm{A}$ is more alexithymic than $\mathrm{B}$ (above 1.64, $P<0.05$ ). Due to the low number of respondents, the frequencies in the categories were converted into percentages. ${ }^{52}$ The estimated standard scores were categorized instead of calculating mean scores, based on the hypothesis that a comparison between the two scales at low and high levels of distributions should be particularly suited for the purpose of this study. A series of chi-square analyses were elaborated to analyze the differential functioning between the two scales in detecting alexithymia in families, family members, and couple contrasts. Chi-square analyses were performed separately for the common factors and the two scale total scores and for the two extreme categories, low and high. The two extreme categories were considered and the middle one was excluded to highlight statistically significant results.

\section{Results \\ Single family analysis}

The alexithymia Rasch estimate standard score for each family member, the family mean standard score, the alexithymia contrast standard score between family members in relation to the TSIA and the TAS-20 total scores, and their common factors are presented in Table 2. The data were obtained for each of the 16 families that participated in the study. For illustrative purposes, only the data related to a single family are introduced herein.

In the selected family, the TSIA detects significantly high alexithymic scores at the total score level for both daughter and father, whereas the mother does not show a significant score. The daughter shows a significantly high score, in particular in relation to factor 1 , whereas the mother shows a significantly low level of alexithymia. Concerning factor 2 , mother and father have significantly high levels of alexithymia, whereas in factor 3 only the father has a significantly high score.

The TAS- 20 provides a very different functioning both at the individual and at the family levels. All members of the family, as regards the total score and, in particular, factors 1 and 2, show significantly low levels of alexithymia. Only for factor 3, although the alexithymia is low, is the result not significant.

It is worth noting that even the distance between levels of alexithymia of family members differs based on the two scales. Considering the TSIA, the daughter proves to have notably greater difficulties in identifying feelings (factor 1) compared with the mother or the father. This is not the case in the TAS-20, where all the family members scored similarly low. The TSIA factor 2 instead shows that the daughter is better able to describe her own feelings compared with her father. The opposite occurs with the TAS-20, where the father considers himself less alexithymic than his daughter.

\section{Alexithymia categories for family, family members, and couples}

As stated, to summarize the data of the 16 families, three categories representing different levels of alexithymia were 


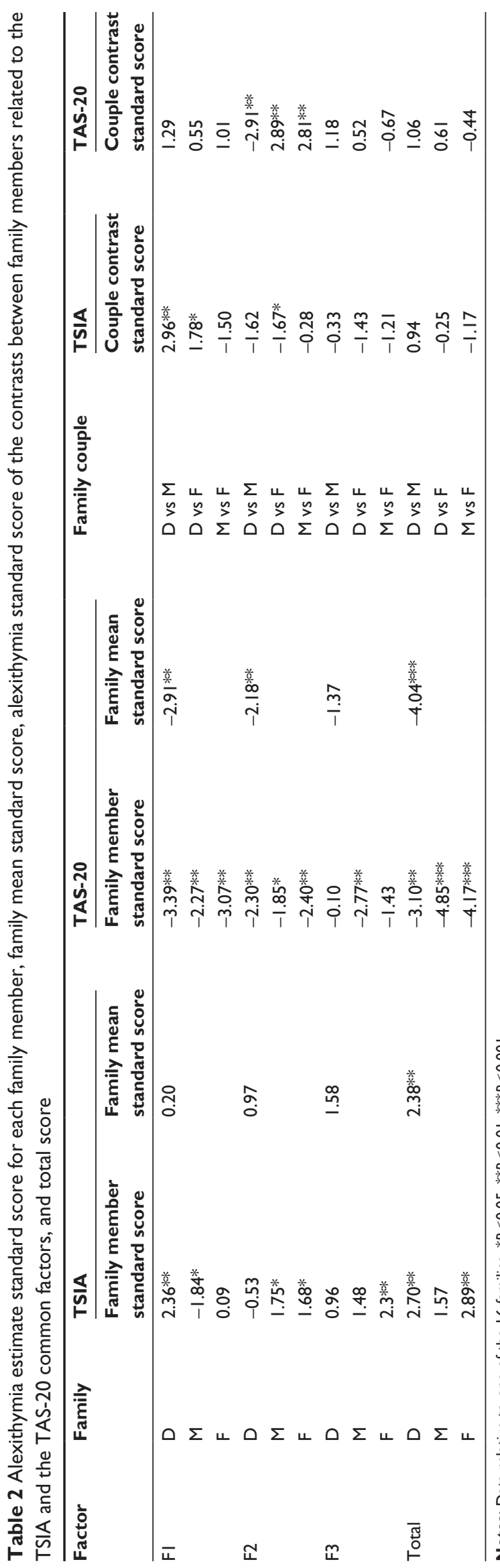

created for the families and separately for daughters, mothers, and fathers. Furthermore, to evaluate the contrasts between alexithymic levels of couples in the family, the same categories were constructed for daughter versus mother, daughter versus father, and mother versus father. The results are illustrated graphically, where the two scales are compared in relation to the three common factors and total score. In Figure 1, the distribution of frequency percentages is associated with the low and high categories for family alexithymia mean standard score; similarly, in Figure 2, the distribution of frequency percentages with the family members; and, in Figure 3, the distribution of frequency percentages relates to the contrasts between family members for the categories low and high. Successively, chi-square analyses were performed to test the statistical significance of the differential functioning between the two scales in relation to the factors and to the total scores.

\section{Family analysis: overall level of family alexithymia}

The data related to the 16 families in Figure 1 indicate that, for all the factors and for the total score, the TAS-20 is characterized by a significantly $(P<0.001)$ larger number of cases (range $18.5 \%-75 \%$ ) than the TSIA (range $0.0 \%-2.5 \%$ ) associated with the low category, which contains the families with average low levels of alexithymia, whereas the TSIA is specified by a significantly $(P<0.001)$ higher number of cases (range 6.25\%-25\%) (except for factor 1) associated with the high category, which includes the families with average high levels of alexithymia. Particularly evident is the differential function of the two scales in relation to factor 1 and to the total score at the low category level and to factor 3 at the high category level.

\section{Family members analysis}

The data from our sample in Figure 2 indicate that, for each family member, for all factors and total scores, the TAS-20 shows a significantly $(P<0.001)$ larger number of cases (range $20 \%-100 \%)$ than the TSIA $(0.0 \%-3.3 \%)$ associated with the low category, whereas the TSIA in general shows a significantly $(P<0.001)$ higher number of cases (6.7\%-53.3\%) than the TAS-20 (range $0.0 \%-8.75 \%$ ) associated with the high category. Considering the low category, a particularly salient difference is between the two scales with regard to factor 1 , factor 3 , and the total score for the daughter, factor 3 and the total score for the mother, and factor 1 for the father. In the high category, the two scales show different functioning, especially in relation to factor 3 


\section{Low category}

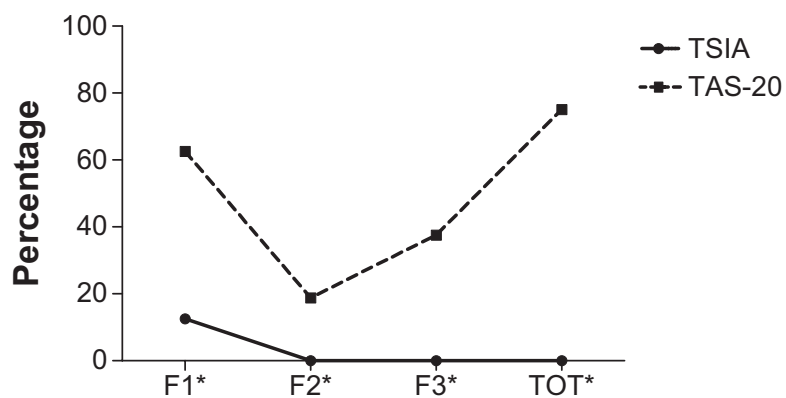

High category

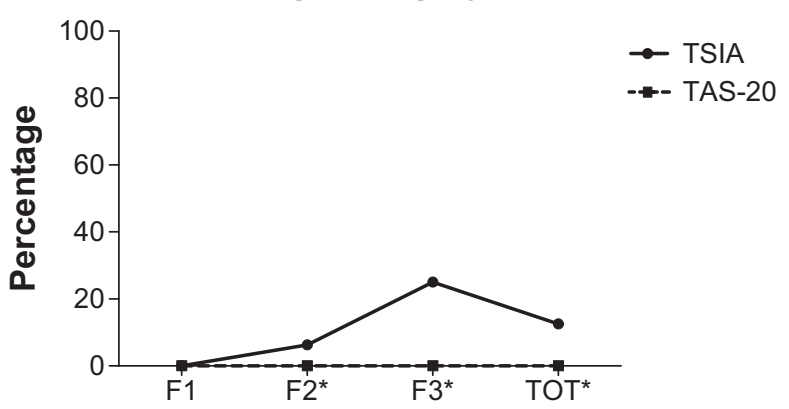

Figure I Family alexithymia mean standard score percentage: a comparison between the TSIA and the TAS-20.

Notes: $*$ TSIA vs TAS- 20 comparison $P<0.05$. Low category: percentage of family obtaining scores below the standard score $-1.64, P<0.05$. High category: percentage of family obtaining scores above the standard score $1.64, P<0.05$.

Abbreviations: TSIA, Toronto Structured Interview for Alexithymia; TAS-20, Toronto Alexithymia Scale; FI, difficulty identifying feelings; F2, difficulty describing feelings; F3, externally oriented thinking; TOT, total score.

for the daughter, factor 2 for the mother, and factor 3 for the father.

\section{Family couple contrasts analysis}

In Figure 3, the distribution of frequency percentages is concerned with the contrasts between members in the family for the low and high categories. The low category contains the cases where the first member in the couple has a significantly lower level of alexithymia than the second member in the couple. On the contrary, the high category includes the cases where the first member in the couple has a significantly higher level of alexithymia than the second one. Once again, the TSIA scale can serve to better illustrate the relations among and between family members compared with the TAS-20, both at the low and the high level.

\section{Discussion}

The prime aim of this study is to examine how the two measuring instruments, the self-report and the clinical interview, developed by the Toronto-based group differ in their ability to detect the presence and intensity of alexithymia within the anorexic patients' families. Despite its widespread use and unquestionably easy application in research domains, the TAS-20 questionnaire has nonetheless revealed some important limits in terms of accuracy and clinical validity, especially when examining psychopathological conditions. ${ }^{27,53}$ Families with anorexic adolescents can prove to be a particularly difficult context that calls for more indepth clinical evaluation of alexithymia rather than the faster, more economical self-report evaluation.

Differently from other studies in literature, latent trait analysis adopted in this research allowed us to examine each subject's alexithymia at a latent level, independently from the content of the items and from other intervening variables, due to the administering set. ${ }^{54}$ Latent trait analysis therefore allows the patients' and parents' alexithymic levels to be compared as detected by the two instruments. Significant discordance was found between the two measures, both in the total as well as in the components of alexithymia, common to both scales (F1-DIF, F2-DDF, F3-EOT).

The clinical instrument detected a greater level of alexithymia compared with the self-report. In particular, in our adult parent sample, alexithymia was higher when based on the structured interview administered by clinicians rather than on the answers the subjects provided themselves, the sole exception being the first factor for the mothers. In such case, the lower scores detected by the two instruments were not significantly different, because mothers generally tend to be aware of their own ability to identify feelings (F1), which is correctly reported in the self-administered test too.

In the young anorexic girls, the TSIA interview, compared with the TAS-20, detected a greater level of alexithymia in the total score and in externally oriented thinking (F3). The results on the difficulties in identifying (F1) and expressing personal feelings (F2) are not as clear. TAS-20 detects a greater number of lower scores compared with TSIA, perhaps due to the girls' lack of awareness or attempt to deny difficulties, ${ }^{55}$ whereas the clinical interview and the self-report have a comparable tendency to score higher in F1 and F2. This is in line with literature, as studies conducted using the TAS-20 self-report alone have succeeded in detecting the significant difficulties anorexic people have in recognizing and verbalizing their emotions (F1, F2). 4,10,14,22

The clinical interview in tandem with the self-report questionnaire adopted in our study, as the authors of the two instruments suggest, ${ }^{35,40}$ have yielded substantially different 

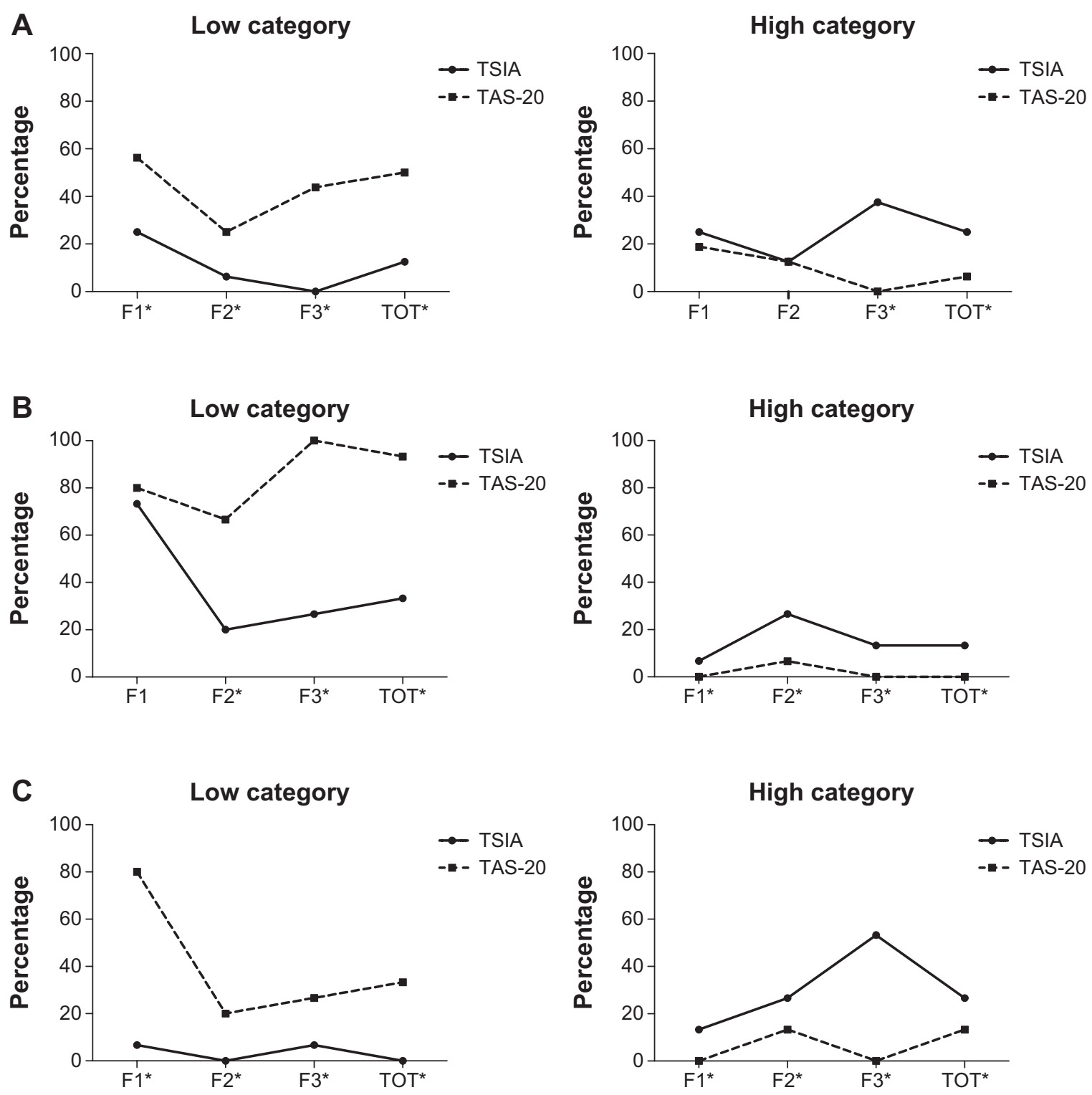

Figure 2 Family members alexithymia standard score percentage: a comparison between the TSIA and the TAS-20 for (A) daughter, (B) mother, and (C) father. Notes: *TSIA vs TAS-20 comparison $P<0.05$. Low category: percentage of $(\mathbf{A})$ daughters, (B) mothers, and $(\mathbf{C})$ fathers obtaining scores below the standard score -1.64 , $P<0.05$. High category: percentage of $(\mathbf{A})$ daughters, $(\mathbf{B})$ mothers, and (C) fathers obtaining scores above the standard score $1.64, P<0.05$.

Abbreviations: TSIA, Toronto Structured Interview for Alexithymia; TAS-20, Toronto Alexithymia Scale; FI, difficulty identifying feelings; F2, difficulty describing feelings; $\mathrm{F} 3$, externally oriented thinking; TOT, total score.

and clinically relevant information on our sample, especially on adults. In fact, a sense of deep crisis and distress for the daughter's condition can lead parents to adopt denial and massive defensive attitudes toward self-administered questionnaires. Otherwise, parents may be so anxious and under such stress that they are unable to answer the questionnaire appropriately, despite their willingness to do so. ${ }^{5}$ Some studies have reported high levels of emotional distress, negative affectivity, depression, and anxiety in the parents of anorexic patients, probably linked to the high levels of alexithymia revealed by self-reports. ${ }^{4,59} \mathrm{We}$ can therefore assume that the interview administered by trained clinicians might be more sensitive to the actual level of alexithymia, as it excludes or attenuates the confounding factors generated by parents' negative affective state, defense mechanisms, varying levels of awareness, and capabilities to reflect on feelings. ${ }^{35,36}$

The study results on the overall level of family alexithymia are worthy of notice. Compared with the TAS-20, the TSIA interview has identified a much higher level of family alexithymia for all of the factors. In the total score, the mean family alexithymia index was very high in some families (13\%) only when using the TSIA measurement, while no families had 
A
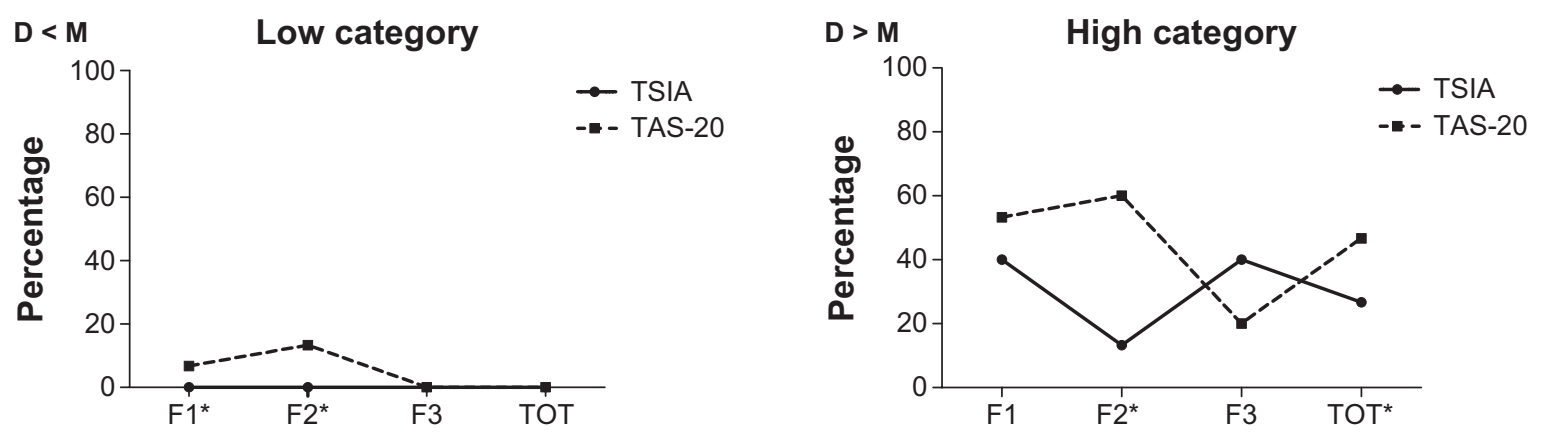

B
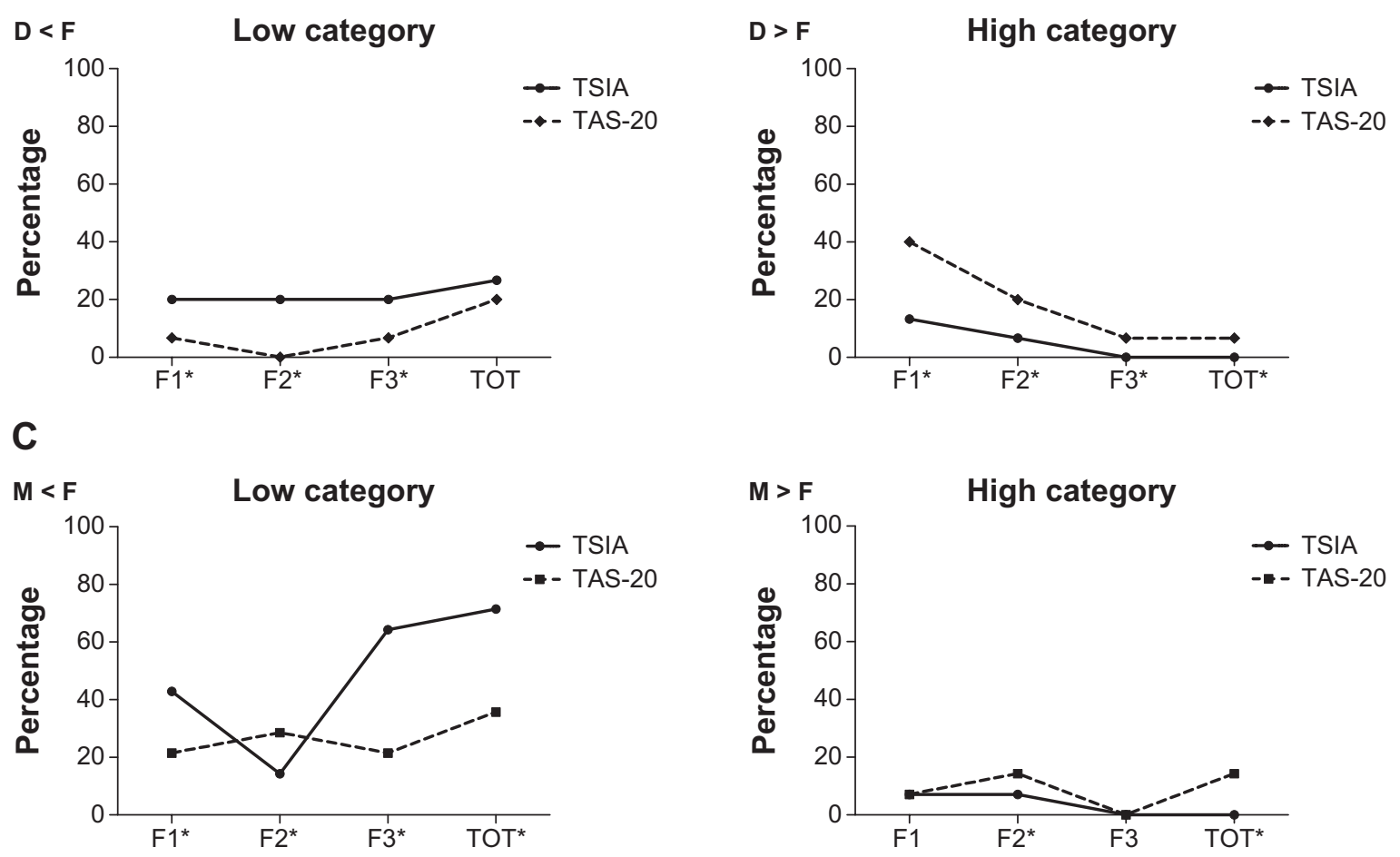

Figure 3 Family couple contrasts alexithymia standard score percentage: a comparison between the TSIA and the TAS-20 for (A) daughter (D) versus mother (M), (B) daughter (D) versus father $(F)$, and $(C)$ mother $(M)$ versus father $(F)$.

Notes: *TSIA vs TAS-20 comparison $P<0.05$. Low category: percentage of contrasts $(\mathbf{A})$ daughter versus mother, $(\mathbf{B})$ daughter versus father, and $(\mathbf{C})$ mother versus father below the standard score $-1.64, \mathbf{P}<0.05$. High category: percentage of contrasts $(\mathbf{A})$ daughter versus mother, $(\mathbf{B})$ daughter versus father, and $(\mathbf{C})$ mother versus father above the standard score I.64, $P<0.05$.

Abbreviations: TSIA, Toronto Structured Interview for Alexithymia; TAS-20, Toronto Alexithymia Scale; FI, difficulty identifying feelings; F2, difficulty describing feelings; F3, externally oriented thinking; TOT, total score.

low alexithymic scores. Paradoxically, the contrary occurs when using TAS-20. No family had high alexithymic scores, with as much as $75 \%$ of the families scoring very low, based on the answers provided by the subjects.

This marked difference between the two measures of family alexithymia is attributable especially to the scores that fathers obtained, which are lower for all of the TAS-20 factors, thus when they were the ones to answer the questionnaire rather than when evaluated by the clinician. This marked difference may highlight the fathers' inability to detect their emotional difficulties, perhaps linked to a defense style founded on external oriented thinking and concrete factual problem solving. ${ }^{5}$

Considering the role of paternal rather than maternal alexithymia is somewhat innovative, compared with the approaches in current studies on adult patients and their parents. ${ }^{4,5}$ Conceivably, this particular way of functioning of fathers may, in part, be a previous salient characteristic of their personality. Within the couple, concrete thinking and cutting off emotions could lead these men to have a 
collusive relationship with more emotional reactive women. The complex dynamics created in this kind of couple may subsequently include young anorexic daughters. Nonetheless, a pivotal point to bear in mind is that the adolescent daughter's life-threatening condition of being is a traumatic factor that often leads such men, who may perhaps already be inclined, to adopt massive defense mechanisms and to freeze their emotions. ${ }^{5}$ This type of paternal response to the daughter's pathology can, in turn, negatively affect the evolution, triggering a vicious circle. Currently, plenty of documented proof illustrates how a lack of communication and emotional communication perceived by the patients in the families, ${ }^{6,956}$ and a greater extent of coldness and hostility, ${ }^{7,9}$ even on the father's part, ${ }^{7,57}$ can play a negative role in eating disorders.

Recent evidence, ${ }^{9}$ in fact, has pointed to the important role of fathers in anorexic adolescents. Male paternal participation proves to be fundamental during the therapeutic process. If paternal warmth and participation have a positive effect on the outcome of the daughter's anorexia, on the contrary, the paternal alexithymia studied herein can imply lesser involvement and therefore negatively impact the quality of family relations and thus the girl's outcome. ${ }^{9}$ We therefore wish to underscore that, under a therapeutic perspective, the father's role and the reciprocal emotional communication among family members need to be tackled. Encouraging the participation and support of fathers, who generally tend to slip away, wishing to remain excluded emotionally and concretely, can prove crucial when trying to modify intrafamily dynamics, which appear to be the key for successful family therapy in adolescent patients with anorexia. ${ }^{1}$

Family relationships are well worth investigating from such a perspective. In the case of alexithymia, the differences in emotional functioning among the various family members were analyzed. The focus was placed primarily on examining how various couples, mother-daughter, father-daughter, and mother-father, differ in terms of alexithymia. Even at this level the TSIA and the TAS-20 differ, especially when considering, within the couples, the father's alexithymia, which turns out to be lower in the self-report and higher in the interview. In considering total alexithymia, the comparison involving the mother and father parental couple is the most striking. Mothers tend to be less alexithymic than fathers. This difference is even more striking from the use of the clinical interview, which minimizes the paternal negation tendency. Total alexithymia, when measured with the TSIA, revealed a wide gap between the two partners in as much as $71 \%$ of the couples, with husbands being more alexithymic than wives. The level of alexithymia between the two partners is therefore comparable only in a very limited number of couples where there is a sort of alexithymic accordance, whereas the opposite case, that of a more alexithymic wife, was not detected in any couple.

Many daughters also have a lower level of alexithymia compared with their father's when measured with the TSIA clinical interview. The exact contrary is true with the TAS-20 self-report, as fathers are more inclined toward negation, whereas, in both self-report and interview, most cases resulted in more alexithymic daughters compared with their mother, whose alexithymia is generally not high.

The aforementioned gap reflects the way different family members experience feelings within the family. It may be a significant clinical index that could influence family relationships ${ }^{58}$ Subsequent studies could explore the value of this index as a discriminant factor in relation to the kind of family functioning, the potential therapeutic alliance with the families, and perhaps the daughter's outcome. In fact, literature considers family relationships to be more relevant than the parents' individual personality and psychopathological traits in predicting the anorexic daughter's alexithymia. ${ }^{4}$ In addition, unhealthy family functioning and affective responsiveness have proved to be connected with the specific risk factors that may trigger anorexia. ${ }^{8}$ Likewise, after the onset of the disorder in the daughter, individual psychological traits of family members and the way they express emotions continue to be interrelated to the clinical severity of anorexia. ${ }^{9,56,59}$

One of the major innovations in our study is the use of the TSIA. To date, no other study has explored the alexithymia of adolescent anorexic patients and their parents using selfreport in tandem with a clinical interview. Results suggest that a clinical interview may be a more sensitive instrument in detecting alexithymia. Compared with the TAS-20, the clinical interview may detect different, and perhaps finer, elements and thus be better at capturing the gap among the different family members' alexithymia. This would justify carrying out the validation process of the TSIA for the Italian adolescent population.

Aside from the aforementioned lack of validation for Italian adolescents, the study's other weaknesses include not having a control group and requiring an expanded adolescent-parent sample. It is important to note that the Rasch modeling approach applied in this study, due to the model's property invariance of comparisons, should substantially overcome the limitation of lacking a comparator group. ${ }^{54}$ Future research should thus be extended to include 
other families within the sample and, whenever possible, the patient's siblings within each family. Presently, this was not possible due to ethical problems and to the family's reluctance to adhere. Future studies could also integrate an evaluation of other psychological aspects (ie, attachment, ${ }^{51}$ depression ${ }^{19,20}$ ) studied not only at the individual level but also within the family system. It may also be interesting to include some outcome measures on adolescent anorexia. The value and the relevance of the alexithymic index at the family level could thus be assessed. In the future, the authors expect to carry out a prolonged observation of the patients' evolution to gain better understanding of whether the variables studied are prognostically significant.

\section{Conclusion}

Results seem to indicate that the clinical interview may be a more sensitive instrument compared with the self-report, allowing data integration on the functioning of adolescent anorexic patients and their parents. The TSIA interview can therefore be an instrument worth validating for the adolescent age group.

Moreover, the study of a couple's functioning in terms of alexithymia has spotlighted a significant alexithymic gap within families, particularly within parental couples, where fathers are noticeably more alexithymic than mothers. Clinical questions have arisen on how useful it may be to give greater weight to family functioning rather than to individual personality traits in predicting the possibility of establishing a therapeutic alliance and thus the outcome of the anorexic adolescent.

\section{Acknowledgments}

The authors warmly thank all of the adolescents and parents who took part in the study, along with all those who have supported us with their precious help.

\section{Disclosure}

The author reports no conflicts of interest in this work.

\section{References}

1. Godart N, Berthoz S, Curt F, et al. A randomized controlled trial of adjunctive family therapy and treatment as usual following inpatient treatment for anorexia nervosa adolescents. PLoS One. 2012;7(1):e28249.

2. Lumley MA, Mader C, Gramzow J, Papineau K. Family factors related to alexithymia characteristics. Psychosom Med. 1996;58(3):211-216.

3. Dahlman K. Affective capacity in mothers of eating disorders patients. Diss Abstr Int B Sci Eng. 1996;56(9):5163-5164.

4. Guttman H, Laporte L. Alexithymia, empathy, and psychological symptoms in a family context. Compr Psychiatry. 2002;43(6):448-455.

5. Espina A. Alexithymia in parents of daughters with eating disorders: its relationships with psychopathological and personality variables. J Psychosom Res. 2003;55(6):553-560.
6. Rodríguez Martín A, Novalbos Ruiz JP, Martínez Nieto JM, Escobar Jiménez L, Castro De Haro AL. Epidemiological study of the influence of family and socioeconomic status in disorders of eating behaviour. Eur J Clin Nutr. 2004;58(6):846-852.

7. Ravi S, Forsberg S, Fitzpatrick K, Lock J. Is there a relationship between parental self-reported psychopathology and symptom severity in adolescents with anorexia nervosa? Eat Disord. 2009;17(1):63-71.

8. Lyke J, Matsen J. Family functioning and risk factors for disordered eating. Eat Behav. 2013;14(4):497-499.

9. Duclos J, Dorard G, Berthoz S, et al. Expressed emotion in anorexia nervosa: what is inside the "black box"? Compr Psychiatry. 2014;55(1): 71-79.

10. Taylor GJ, Parker JD, Bagby RM, Bourke MP. Relationships between alexithymia and psychological characteristics associated with eating disorders. J Psychosom Res. 1996;41(6):561-568.

11. Harrison A, Sullivan S, Tchanturia K, Treasure J. Emotion recognition and regulation in anorexia nervosa. Clin Psychol Psychother. 2009;16(4):348-356.

12. Bomba M, Corbetta F, Gambera A, et al. Heart rate variability in adolescents with functional hypothalamic amenorrhea and anorexia nervosa. Psychiatry Res. 2014;215(2):406-409.

13. Conti E, Tremolizzo L, Bomba M, et al. Reduced fasting plasma levels of diazepam-binding inhibitor in adolescents with anorexia nervosa. Int J Eat Disord. 2013;46(6):626-629.

14. Speranza M, Loas G, Wallier J, Corcos M. Predictive value of alexithymia in patients with eating disorders: a 3-year prospective study. J Psychosom Res. 2007;63(4):365-371.

15. Zonnevijlle-Bender MJ, van Goozen SH, Cohen-Kettenis PT, van Elburg A, van Engeland H. Do adolescent anorexia nervosa patients have deficits in emotional functioning? Eur Child Adolesc Psychiatry. 2002;11(1):38-42.

16. Zonnevylle-Bender MJ, van Goozen SH, Cohen-Kettenis PT, van Elburg TA, van Engeland H. Emotional functioning in adolescent anorexia nervosa patients - a controlled study. Eur Child Adolesc Psychiatry. 2004;13(1):28-34.

17. Zonnevylle-Bender MJ, van Goozen SH, Cohen-Kettenis PT, et al. Emotional functioning in anorexia nervosa patients: adolescents compared to adults. Depress Anxiety. 2004;19(1):35-42.

18. Lulé D, Schulze UM, Bauer K, et al. Anorexia nervosa and its relation to depression, anxiety, alexithymia and emotional processing deficits. Eat Weight Disord. 2014;19(2):209-216.

19. Bomba M, Corbetta F, Bonini L, et al. Psychopathological traits of adolescents with functional hypothalamic amenorrhea: a comparison with anorexia nervosa. Eat Weight Disord. 2014;19(1):41-48.

20. Corcos M, Guilbaud O, Speranza M, et al. Alexithymia and depression in eating disorders. Psychiatry Res. 2000;93(3):263-266.

21. Speranza M, Stéphan P, Corcos M, et al. [Relationships between alexithymia, depression and interpersonal dependency in addictive subjects]. Ann Med Interne (Paris). 2003;154(Spec No. 1):S65-S75. French.

22. Speranza M, Corcos M, Loas G, et al. Depressive personality dimensions and alexithymia in eating disorders. Psychiatry Res. 2005;135(2): 153-163.

23. Montebarocci O, Codispoti M, Surcinelli P, Franzoni E, Baldaro B, Rossi N. Alexithymia in female patients with eating disorders. Eat Weight Disord. 2006;11(1):14-21.

24. Parling T, Mortazavi M, Ghaderi A. Alexithymia and emotional awareness in anorexia nervosa: time for a shift in the measurement of the concept? Eat Behav. 2010;11(4):205-210.

25. Rozenstein MH, Latzer Y, Stein D, Eviatar Z. Perception of emotion and bilateral advantage in women with eating disorders, their healthy sisters, and nonrelated healthy controls. J Affect Disord. 2011; 134(1-3):386-395.

26. Lumley MA. Alexithymia and negative emotional conditions. J Psychosom Res. 2000;49(1):51-54.

27. Lumley MA, Neely LC, Burger AJ. The assessment of alexithymia in medical settings: implications for understanding and treating health problems. J Pers Assess. 2007;89(3):230-246. 
28. Caretti V, La Barbera D, eds. Alessitimia. Valutazione e trattamento. Rome, Italy: Astrolabio; 2005.

29. Taylor GJ, Bagby RM. New trends in alexithymia research. Psychother Psychosom. 2004;73(2):68-77.

30. Guilbaud O, Berthoz S, Dupont ME, Corcos M. Alexithymie et troubles psychosomatiques. [Alexithymia and psychosomatic disorders]. Psychiatrie. 2009;37-400-D-20. French.

31. Berthoz S, Perdereau F, Godart N, Corcos M, Haviland MG. Observerand self-rated alexithymia in eating disorder patients: levels and correspondence among three measures. $J$ Psychosom Res. 2007;62(3): 341-347.

32. Haviland MG, Warren WL, Riggs ML. An observer scale to measure alexithymia. Psychosomatics. 2000;41:385-392.

33. Bagby RM, Parker JD, Taylor GJ. The twenty-item Toronto Alexithymia Scale-I. Item selection and cross-validation of the factor structure. J Psychosom Res. 1994;38(1):23-32.

34. Bagby RM, Taylor GJ, Parker JD. The Twenty-item Toronto Alexithymia Scale-II. Convergent, discriminant, and concurrent validity. $J$ Psychosom Res. 1994;38(1):33-40.

35. Bagby RM, Taylor GJ, Parker JD, Dickens SE. The development of the Toronto Structured Interview for Alexithymia: item selection, factor structure, reliability and concurrent validity. Psychother Psychosom. 2006;75(1):25-39.

36. Tremolizzo L, Conti E, Bomba M, et al. Decreased whole-blood global DNA methylation is related to serum hormones in anorexia nervosa adolescents. World J Biol Psychiatry. 2014;15(4):327-333.

37. Bomba M, Riva A, Veggo F, et al. Impact of speed and magnitude of weight loss on the development of brain trophic changes in adolescents with anorexia nervosa: a case control study. Ital J Pediatr. 2013; 39:14.

38. Bressi C, Taylor G, Parker J, et al. Cross validation of the factor structure of the 20 -item Toronto Alexithymia Scale: an Italian multicenter study. J Psychosom Res. 1996;41(6):551-559.

39. Taylor GJ, Bagby RM. Measurement of alexithymia. Recommendations for clinical practice and future research. Psychiatr Clin North Am. 1988;11(3):351-366.

40. Taylor GJ, Bagby RM. New trends in alexithymia research. Psychother Psychosom. 2004;73(2):68-77.

41. Caretti V, Porcelli P, Solano L, Schimmenti A, Bagby RM, Taylor GJ. Reliability and validity of the Toronto Structured Interview for Alexithymia in a mixed clinical and nonclinical sample from Italy. Psychiatry Res. 2011;187(3):432-436.

42. Garner DM. EDI-3 Eating Disorder Inventory-3. Adattamento Italiano. Florence, Italy: Giunti Organizzazioni Speciali; 2008

43. Kaufman J, Birmaher B, Brent DA, Ryan ND, Rao U. K-SADS-PL. J Am Acad Child Adolesc Psychiatry. 2000;39(10):1208.

44. Rasch G. Probabilistic Models for Some Intelligence and Attainment Tests. Chicago, IL: The University of Chicago Press; 1960.
45. Andrich DA. Rating formulation for ordered response categories. Psychometrika. 1978;43:561-573.

46. Linacre JM. A user's guide to WINSTEPS MINISTEP Rasch-Model computer programs (version 3.81.0.0) [webpage on the Internet]. Available from: http://www.winsteps.com. Accessed September 2, 2014.

47. Mannarini S. A method for the definition of a self-awareness dimension with clinical subjects: a latent trait analysis. Behav Res Methods. 2009;41(4):1029-1037.

48. Mannarini S. Assessing the Rosenberg Self-Esteem Scale dimensionality and items functioning in relation to self-efficacy and attachment styles. TPM Test Psychom Methodol Appl Psychol. 2010;17(4):229-242.

49. Mannarini S, Polimeni S, Shams M, Giacobbo M. Assessing negative and positive mood states: the identification of a short form of the POMS scale in Italian oncology outpatients. TPM Test Psychom Methodol Appl Psychol. 2012;19(2):135-145.

50. Mannarini S, Boffo M, Bertucci V, Andrisani A, Ambrosini GA. Raschbased dimension of delivery experience: spontaneous vs medically assisted conception. J Clin Nurs. 2013;22(17-18):2404-2416.

51. Mannarini S, Boffo M. The relevance of security: a latent domain of attachment relationships. Scand J Psychol. 2014;55:53-59.

52. Koehler KJ, Larntz K. An empirical investigation of goodness-of-fit statistics for sparse multinomials. J Am Stat Assoc. 1980;75:336-344.

53. Leising D, Grande T, Faber R. The Toronto Alexithymia Scale (TAS-20): a measure of general psychological distress. J Res Pers. 2009;43(4): 707-710.

54. Cristante F, Mannarini S. Misurare in Psicologia. Il Modello di Rasch. Bari, Italy: Laterza; 2004.

55. Corbetta F, Bomba M, Tremolizzo L, Neri F, Nacinovich R. The use of the Psychodynamic Diagnostic Manual (PDM) in child and adolescent psychiatry: application in a sample of adolescents affected by anorexia nervosa. Giornale di Neuropsichiatria dell'Età Evolutiva. 2014;34:29-38.

56. Rommel D, Nandrino JL, Antoine P, Dodin V. Emotional differentiation and parental bonding in inpatients suffering from eating disorders. Br J Clin Psychol. 2013;52(2):215-229.

57. Le Grange D, Lock J, Loeb K, Nicholls D. Academy for eating disorders position paper: the role of the family in eating disorders. Int $J$ Eat Disord. 2010;43(1):1-5.

58. Inslegers R, Vanheule S, Meganck R, Debaere V, Trenson E, Desmet M. Interpersonal problems and cognitive characteristics of interpersonal representations in alexithymia: a study using a self-report and interview-based measure of alexithymia. J Nerv Ment Dis. 2012;200(7):607-613.

59. Fornari V, Wlodarczyk-Bisaga K, Matthews M, Sandberg D, Mandel FS, Katz JL. Perception of family functioning and depressive symptomatology in individuals with anorexia nervosa or bulimia nervosa. Compr Psychiatry. 1999;40(6):434-441.
Neuropsychiatric Disease and Treatment

\section{Publish your work in this journal}

Neuropsychiatric Disease and Treatment is an international, peerreviewed journal of clinical therapeutics and pharmacology focusing on concise rapid reporting of clinical or pre-clinical studies on a range of neuropsychiatric and neurological disorders. This journal is indexed on PubMed Central, the 'PsycINFO' database and CAS,

\section{Dovepress}

and is the official journal of The International Neuropsychiatric Association (INA). The manuscript management system is completely online and includes a very quick and fair peer-review system, which is all easy to use. Visit http://www.dovepress.com/testimonials.php to read real quotes from published authors. 